\title{
Research Article \\ On the Speed of Spread for Fractional Reaction-Diffusion Equations
}

\author{
Hans Engler \\ Department of Mathematics, Georgetown University, Box 571233, Washington, DC 20057, USA \\ Correspondence should be addressed to Hans Engler, engler@georgetown.edu
}

Received 12 August 2009; Revised 12 October 2009; Accepted 25 October 2009

Academic Editor: Om Agrawal

Copyright (c) 2010 Hans Engler. This is an open access article distributed under the Creative Commons Attribution License, which permits unrestricted use, distribution, and reproduction in any medium, provided the original work is properly cited.

The fractional reaction diffusion equation $\partial_{t} u+A u=g(u)$ is discussed, where $A$ is a fractional differential operator on $\mathbb{R}$ of order $\alpha \in(0,2)$, the $C^{1}$ function $g$ vanishes at $\zeta=0$ and $\zeta=1$, and either $g \geq 0$ on $(0,1)$ or $g<0$ near $\zeta=0$. In the case of nonnegative $g$, it is shown that solutions with initial support on the positive half axis spread into the left half axis with unbounded speed if $g(\zeta)$ satisfies some weak growth condition near $\zeta=0$ in the case $\alpha>1$, or if $g$ is merely positive on a sufficiently large interval near $\zeta=1$ in the case $\alpha<1$. On the other hand, it shown that solutions spread with finite speed if $g^{\prime}(0)<0$. The proofs use comparison arguments and a suitable family of travelling wave solutions.

\section{Introduction}

The scalar reaction-diffusion equation

$$
\partial_{t} u(x, t)-\partial_{x}^{2} u(x, t)=g(u(x, t))
$$

has been the subject of much study, beginning with the celebrated paper [1]. The authors of [1] proposed this equation, with $g$ being positive and concave on $(0,1)$ such that $g(0)=$ $g(1)=0$, as a model for a population that undergoes logistic growth and Brownian diffusion. If $u(x, 0)=H(x)$, the Heaviside function, and $g(u)=u-u^{2}$, the equation in fact has an exact probabilistic interpretation, given in [2]. Consider a population of particles that undergo independent Brownian motion and branching processes, with each child particle again following the same behavior. Then $u(x, t)$ is the probability that there is a particle to the left of position $x$ at time $t$, assuming that there was exactly one particle at position $x=0$ at time $t=0$. Equation (1.1) also can be derived heuristically for the mean behavior of an 
interacting particle process in which two types of particles (call them $A$ - and $B$-particles) simultaneously undergo Brownian diffusion and conversion reactions

$$
A+B \longrightarrow 2 A, \quad A+B \longrightarrow 2 B
$$

with suitable reaction rates. Then the volume density fraction $u(x, t)$ of $A$-particles in the hydrodynamic limit of large particle numbers per unit volume formally satisfies (1.1) with $g(u)=c u(1-u)$ where $c$ depends on the reaction rates in (1.2); see [3] for a discussion of the underlying limit procedure and an exact connection to a stochastic version of (1.1). If instead the conversion reactions are

$$
2 A+B \longrightarrow 3 A, \quad 2 A+B \longrightarrow A+2 B
$$

then the equation for $u$ becomes (1.1) with $g(u)=c u^{2}(1-u)$. Other polynomial reaction terms $g$ occur in similar ways.

For (1.1) with $g(u)=u(1-u)$, it is known that solutions approach a wave profile $\psi$ in the sense that

$$
u(x+m(t), t) \longrightarrow \psi(x) \quad(t \longrightarrow \infty)
$$

where $m(t)$ is the median, $u(m(t), t)=1 / 2$. It turns out that $m(t)=c^{*} t+O(\log t)$ for a suitable asymptotic finite wave speed $c^{*}$. Larger asymptotic speeds are only possible if the initial data are supported on $\mathbb{R}$. A more general result, given in [4], implies that there is a critical speed $c^{*}$ such that for fairly general initial data $u(\cdot, 0)$ that are nonnegative and supported on $(0, \infty)$,

$$
\limsup _{t \rightarrow \infty} \sup _{x<-c t} u(x, t)=0
$$

whenever $c>c^{*}$ and

$$
\liminf _{t \rightarrow \infty} \inf _{x<-c t} u(x, t)=1
$$

whenever $c<c^{*}$. If $u$ is interpreted as the density of a quantity whose spread is governed by (1.1), a runner may escape from it by running to $-\infty$ at a speed $c>c^{*}$, but this quantity will catch up with and engulf it if its speed is $c<c^{*}$. In this sense, solutions of the (1.1) exhibit finite speed of spread.

Equation (1.1) was also derived in [5] to describe antiphase domain coarsening in alloys. In this situation, $g(0)=g(1)=g\left(u^{*}\right)=0$ for some $u^{*} \in(0,1)$, and $g<0$ on $\left(0, u^{*}\right)$, and $g>0$ on $\left(u^{*}, 1\right)$. In this case there exists exactly one wave speed $c^{*}$ with associated wave profile. In particular, (1.5) and (1.6) still hold for this $c^{*}$. The first of the two cases (the KPP case) corresponds to "pulled" fronts (the state $u=0$ is unstable) while the second case (the Allen-Cahn case) results in a "pushed" front (the state $u=0$ is stable). More on these two fundamentally different situations may be found in [6] and the references given there. A vast range of applications leading to related models is discussed in [7]. 
The purpose of this note is a study of the fractional reaction-diffusion equation:

$$
\partial_{t} u(x, t)+A u(x, t)=g(u(x, t)) .
$$

Here $A=A_{\alpha \rho}$ is a pseudodifferential operator with symbol $p=p_{\alpha \rho}$ that is homogeneous of degree $\alpha \in(0,2]$, such that $p(-\lambda)=\overline{p(\lambda)}$ and $|p(1)|=1$. Following the presentation in [8], we write $p$ in the Riesz-Feller form:

$$
p(\lambda)=e^{-i(\pi / 2) \operatorname{sign}(\lambda) \rho}|\lambda|^{\alpha},
$$

where the skewness parameter $\rho$ must also satisfy $|\rho| \leq \min \{\alpha, 2-\alpha\}$. The operator $A$ is the infinitesimal generator of a stable Lévy process, that is, a continuous time stochastic process that has cádlág paths and independent stationary increments with stable distributions; see [9]. Paths of such a process must have jumps, and the variance of the displacement must be infinite.

For $\rho=0$ we obtain fractional powers of the usual negative one-dimensional Laplacian, abbreviated often by $(-\Delta)^{\alpha / 2}$. There are various real variable representations of such operators, for example, as singular integral operators or as limits of suitable difference operators; see [8]. In the special case where $\alpha=1$ and $-1 \leq \rho \leq 1$, there is the representation

$$
A_{1, \rho} u(x)=\frac{d}{d x}\left(\cos \frac{\rho \pi}{2} \mathscr{H} u(x)+\sin \frac{\rho \pi}{2} u(x)\right)
$$

where $\mathscr{d} u$ is the Hilbert transform of $u$. In particular, for $\rho= \pm 1$, this is an ordinary first-order derivative, not a fractional derivative.

The function $g$ is always assumed to satisfy $g(0)=g(1)=0$. We are interested in both the KPP-case, that is, $g(\zeta) \geq 0$ for $0<\zeta<1$, and the Allen-Cahn case, that is, $g(\zeta)<0$ for $\zeta$ near 0 and $g(\zeta)>0$ for $\zeta$ near 1 .

The equation occurs in the heuristic hydrodynamic limit of interacting particle populations in which conversion reactions such as (1.2) or (1.3) occur together with motion by a stable Lévy process. It has been proposed in, for example, [10-15]. It should be noted that the term "anomalous diffusion" is also used for situations in which the first-order time derivative is replaced by a fractional order derivative; see [8] where the present case of a first-order time derivative is called space-fractional diffusion. Another generalization of (1.1) consists in allowing time delays; see [16]. These further generalizations will not be discussed here.

There is strong evidence that (1.7) does not admit traveling wave solutions if $0<\alpha<2$ and $g$ is positive and concave on $(0,1)$. Rather, numerical results in [11,17] suggest that for initial data that are supported on the positive half axis and increase there from 0 to 1 , the median satisfies $m(t) \sim-e^{c t}$ for some $c>0$. In [18], the estimates

$$
\limsup _{t \rightarrow \infty} \sup _{x<-e^{c t}} u(x, t)=0, \quad \liminf _{t \rightarrow \infty} \inf _{x>-e^{d t}} u(x, t)=1
$$

are shown to hold for such initial data whenever $c>c^{*}>d>0$, where $c^{*}=g^{\prime}(0) / \alpha$. Thus the support of a solution grows asymptotically like an exponential. For the case where $g<0$ on 
some interval $\left(0, u^{*}\right)$, the results in $[14,19]$ suggest on the other hand that there exist wave profile solutions that move with constant speed, although no rigorous proofs are given there.

The main results of this note are concerned with the existence and nonexistence of a finite speed of spread and take the form (1.5) and (1.6). It is shown that for a large class of right-hand sides $g$ that are nonnegative on $(0,1)$, the speed of spread is infinite; that is, the estimate (1.6) holds for all positive speeds $c$. It is not necessary to assume that $g^{\prime}(0)>0$, and if $\alpha<1$, one does not even have to assume that $g$ is strictly positive on $(0,1)$. On the other hand, if $g^{\prime}(0)<0$ and therefore $g$ is negative near 0 , then it will be shown that there exists a finite speed of spread; that is, (1.5) holds for some finite positive $c$. These results are stated and proven in Section 3. Some basic existence and comparison results for (1.7) are sketched in Section 4.

To prove these results, comparison arguments are employed which follow from integral representations of solutions of (1.7) and which are therefore extensions of similar arguments for the study of (1.1). The challenge then is to come up with suitable comparison solutions. Since (1.7) is nonlocal in nature, techniques from ordinary differential equations cannot be employed to construct such solutions. Instead, in this paper a set of travelling wave solutions is used that comes directly from the fundamental solution of the linear problem (see (2.1) below). These solutions are discussed in Section 2 and may be of independent interest.

\section{A Class of Travelling Wave Solutions}

In this section, it will be shown that fundamental solutions of fractional diffusion equations (without reaction terms) lead to traveling wave solutions $u(x, t)=U(x+c t)$ of (1.7), for suitable functions $g$. A two-parameter family will be constructed for each possible choice of $\alpha$ and $\rho$, one parameter being the speed $c$. The main contribution of this section is the characterization of the nonlinear function $g$ that is required to make the equation hold. Throughout this section, let $\alpha \in(0,2)$ and $|\rho|<\min \{\alpha, 2-\alpha\}$.

Consider the "free" equation

$$
\partial_{t} u(x, t)+A_{\alpha \rho} u(x, t)=0,
$$

where $A_{\alpha \rho}$ is the pseudodifferential operator with symbol $p_{\alpha \rho}$ defined in (1.8). It is known that (2.1) has the fundamental solution

$$
(x, t) \longmapsto W_{\alpha \rho}(x, t)=t^{-1 / \alpha} f_{\alpha \rho}\left(x t^{-1 / \alpha}\right)
$$

with initial data $W_{\alpha \rho}(x, 0)=\delta_{0}(x)$, the delta distribution; see [8]. In particular, $W_{\alpha \rho}(x, 1)=$ $f_{\alpha \rho}(x)$. Here $f_{\alpha \rho}$ is the probability density function of a stable distribution; see [20, 21]. There are many ways to parametrize such distributions. For $\alpha \notin\{0,1,2\}$ the version used here corresponds to form (B) in [21] with the same choice of $\alpha$, scale parameter $\gamma=1$, location parameter $\delta=0$, and skewness parameter:

$$
\beta= \pm \frac{\rho}{\min \{\alpha, 2-\alpha\}}
$$


where the sign is positive if $0<\alpha<1$ and negative if $1<\alpha<2$. For $\alpha=1$, the form used here corresponds to form (C) in [21] with $\beta=\rho, \gamma=1, \delta=0$.

There is also the special solution

$$
(x, t) \longmapsto V_{\alpha \rho}(x, t)=F_{\alpha \rho}\left(x t^{-1 / \alpha}\right),
$$

where $F_{\alpha \rho}(x)=\int_{-\infty}^{x} f_{\alpha \rho}(s) d s$ is a cumulative distribution function. Then $V_{\alpha \rho}$ solves (2.1) with initial data $V_{\alpha \rho}(x, 0)=H(x)$, the Heaviside function. The equations hold in the sense of distributions, and the initial data are attained in this sense. It is known that $f_{\alpha \rho}$ is positive, infinitely differentiable, and unimodal. Also, as $x \rightarrow \infty$, there are expansions

$$
\begin{gathered}
1-F_{\alpha \rho}(x) \sim \sum_{j \geq 1} c_{j \alpha \rho} x^{-j \alpha}, \\
f_{\alpha \rho}(x) \sim \sum_{j \geq 1} \widetilde{c}_{j \alpha \rho} x^{-1-j \alpha},
\end{gathered}
$$

and as $x \rightarrow-\infty$

$$
\begin{gathered}
F_{\alpha \rho}(x) \sim \sum_{j \geq 1} d_{j \alpha \rho}(-x)^{-j \alpha} \\
f_{\alpha \rho}(x) \sim \sum_{j \geq 1} \tilde{d}_{j \alpha \rho}(-x)^{-1-j \alpha} .
\end{gathered}
$$

These are convergent expansions if $0<\alpha<1$ and asymptotic expansions if $1<\alpha<2$; see [8].

For the remainder of this section, we suppress the subscripts $\alpha$ and $\rho$ in most formulae that involve $A_{\alpha \rho}, f_{\alpha \rho}$, and $F_{\alpha \rho}$. For fixed $c \in \mathbb{R}$ and $\tau>0$ we consider the function

$$
U_{\tau}(\xi)=F\left(\xi \tau^{-1 / \alpha}\right)
$$

Set $u_{c \tau}(x, t)=U_{\tau}(x+c t)$, then

$$
\begin{aligned}
A u_{c \tau}(x, t) & =-\partial_{\tau} F\left((x+c t) \tau^{-1 / \alpha}\right) \\
& =\frac{1}{\alpha}\left((x+c t) \tau^{-1 / \alpha-1}\right) f\left((x+c t) \tau^{-1 / \alpha}\right) \\
\partial_{t} u_{c \tau}(x, t) & =c \tau^{-1 / \alpha} f\left((x+c t) \tau^{-1 / \alpha}\right),
\end{aligned}
$$

and therefore

$$
\partial_{t} u_{c \tau}(x, t)+A u_{c \tau}(x, t)=\left(\frac{1}{\alpha}\left((x+c t) \tau^{-1 / \alpha-1}\right)+c \tau^{-1 / \alpha}\right) f\left((x+c t) \tau^{-1 / \alpha}\right) .
$$

Now $(x+c t) \tau^{-1 / \alpha}=F^{-1}\left(u_{c \tau}(x, t)\right)$ and consequently

$$
\partial_{t} u_{c \tau}(x, t)+A u_{c \tau}(x, t)=c \tau^{-1 / \alpha} g_{0}\left(u_{c \tau}(x, t)\right)+\frac{1}{\alpha \tau} g_{1}\left(u_{c \tau}(x, t)\right)
$$


with

$$
g_{0}(\zeta)=f\left(F^{-1}(\zeta)\right), \quad g_{1}(\zeta)=F^{-1}(\zeta) f\left(F^{-1}(\zeta)\right)
$$

Equation (2.10) is of the form (1.7), with $g(\zeta)=c \tau^{-1 / \alpha} g_{0}(\zeta)+1 /(\alpha \tau) g_{1}(\zeta)$.

In the case $\alpha=1$ and $-1<\rho<1$, everything is explicit. Let $\kappa=\cos (\pi \rho) / 2$ and $\sigma=\sin (\pi \rho) / 2$. Then by results in [21],

$$
\begin{aligned}
F(x) & =\frac{1}{2}+\frac{1}{\pi} \arctan \frac{x-\sigma}{\kappa}, \\
F^{-1}(\zeta) & =\sigma-\kappa \cot (\pi \zeta) \\
f(x) & =\frac{1}{\pi \kappa\left(1+(x-\sigma)^{2} / \kappa^{2}\right)} \\
g_{0}(\zeta) & =\frac{1}{\kappa \pi} \sin ^{2}(\pi \zeta) \\
g_{1}(\zeta) & =\frac{\sigma}{\pi \mathcal{K}} \sin ^{2}(\pi \zeta)-\frac{1}{\pi} \cos (\pi \zeta) \sin (\pi \zeta)
\end{aligned}
$$

It remains to characterize the functions $g_{0}, g_{1}$ in the general case.

Proposition 2.1. Let $0<\alpha<2,|\rho|<\min \{\alpha, 2-\alpha\}$. The functions $g_{0}, g_{1}$ have the following properties.

(a) $g_{0}$ and $g_{1}$ are infinitely differentiable on $(0,1)$.

(b) The function $g_{0}$ is positive on $(0,1)$. The function $g_{1}$ is negative on $\left(0, F_{\alpha \rho}(0)\right)$ and positive on $\left(F_{\alpha \rho}(0), 1\right)$. The function

$$
\zeta \longmapsto c \tau^{-1 / \alpha} g_{0}(\zeta)+\frac{1}{\alpha \tau} g_{1}(\zeta)
$$

is negative on $\left(0, u^{*}\right)$ and positive on $\left(u^{*}, 1\right)$, where $u^{*}=F_{\alpha \rho}\left(-c \alpha \tau^{-1 / \alpha+1}\right)$.

(c) As $\zeta \downarrow 0, g_{0}(\zeta)=O\left(\zeta^{1+1 / \alpha}\right)$ and $g_{0}(1-\zeta)=O\left(\zeta^{1+1 / \alpha}\right)$.

(d) As $\zeta \downarrow 0, g_{1}(\zeta)=-\alpha \zeta+O\left(\zeta^{1+1 / \alpha}\right)$. As $\zeta \uparrow 1, g_{1}(\zeta)=\alpha(1-\zeta)+O\left((1-\zeta)^{1+1 / \alpha}\right)$.

(e) The functions $g_{0}$ and $g_{1}$ can be represented as

$$
\begin{aligned}
& g_{0}(\zeta)=\frac{d}{d \zeta} \int_{-\infty}^{F^{-1}(\zeta)} f^{2}(s) d s \\
& g_{1}(\zeta)=\frac{d}{d \zeta} \int_{-\infty}^{F^{-1}(\zeta)} s f^{2}(s) d s .
\end{aligned}
$$

Proof. Property (a) follows since $f$ and $F$ together with its inverse are infinitely differentiable. Property (b) is obvious. Properties (c) and (d) follow from the asymptotic expansions (2.5) and (2.6). Finally (e) can be checked by differentiation. 
Property (e) will not be used in what follows. It should be noted that $g_{0}$ and $g_{1}$ are of class $C^{1}$ on $[0,1]$ but are not infinitely differentiable at the interval endpoints, except if $\alpha=1$. Clearly $g_{0}$ and $g_{1}$ do not depend on $c$ or $\tau$. We are therefore free to form fairly arbitrary linear combinations of $g_{0}$ and $g_{1}$ by choosing $c$ and $\tau$.

The construction provides travelling wave solutions for (1.7) for a special class of functions for which $g \in C^{1}([0,1]), g(0)=g\left(u^{*}\right)=g(1)$ for some $u^{*} \in(0,1)$, and $g^{\prime}(0)<$ $0, g^{\prime}\left(u^{*}\right)>0, g^{\prime}(1)<0$. This suggests that (1.7) possesses travelling wave solutions for more general functions $g$ with these properties.

If the same construction is attempted for the case $\alpha=2$, it turns out that $g_{0}$ and $g_{1}$ are merely continuous on $[0,1]$, with derivatives that have logarithmic singularities near $\zeta=0$ and $\zeta=1$. Therefore the arguments in the next section cannot be extended to the case $\alpha=2$, and indeed the results of the next section do not hold in that case.

\section{Results on the Speed of Spread}

This section contains the main results of this paper. As before, the operator $A$ has symbol (1.8) with $0<\alpha<2$ and $|\rho|<\min \{\alpha, 2-\alpha\}$. We always assume that $u$ is a solution of (1.7) and that $g \in C^{1}([0,1], \mathbb{R})$ with $g(0)=g(1)=0$. Initial data $u_{0}$ will be assumed to satisfy

$$
u_{0} \in C(\mathbb{R}, \mathbb{R}), \quad 0 \leq u_{0}(x) \leq 1, \quad \lim _{x \rightarrow \infty} u_{0}(x)=1, \quad \operatorname{supp}\left(u_{0}\right) \subset[0, \infty) .
$$

The results in Section 4 then imply that (1.7) has a unique mild solution $u$ that exists for all $x \in \mathbb{R}, t>0$, and this solution satisfies $0 \leq u(x, t) \leq 1$ for all $(x, t)$. The notation of that section will also be used here.

We first discuss the case where $g \geq 0$ on $(0,1)$. The main result in this case is the following.

Theorem 3.1. Let $u$ be the solution of (1.7) with $u_{0}$ satisfying (3.1).

(a) Let $\alpha>1$. Assume that $g>0$ on $(0,1)$ and that there are $0<\gamma<\alpha /(\alpha-1), c_{0}>0$ such that for all $\zeta \in[0,1 / 2]$

$$
g(\zeta) \geq c_{0} \zeta^{\gamma}
$$

Then for all $c>0$

$$
\liminf _{t \rightarrow \infty} \inf _{x \geq-c t} u(x, t)=1
$$

(b) Let $\alpha=1$. Assume that $g>0$ on $(0,1)$. Then for all $c>0$

$$
\liminf _{t \rightarrow \infty} \inf _{x \geq-c t} u(x, t)=1 .
$$

(c) Let $\alpha<1$. Assume that $g \geq 0$ on $(0,1)$ and $g(\zeta)>0$ for $\zeta \in[(\alpha-\rho) / 2 \alpha, 1)$. Then for all $c>0$

$$
\liminf _{t \rightarrow \infty} \inf _{x \geq-c t} u(x, t)=1
$$


The result shows that the speed of spread is unbounded (i.e., (1.6) holds for all $c>0$ ), and it exhibits different mechanisms for this phenomenon. Recall that in the interpretation of [1], the function $g$ is responsible for the growth of a substance whose density is given by $u$, while $A$ describes the spread of this substance. If $\alpha \in(0,2)$, the substance spreads with a jump process, not with Brownian diffusion, and jumps of magnitude exceeding $M$ occur with a probability that is $O\left(M^{-\alpha}\right)$ for large $M$. If $\alpha>1$, the mean jump distance is still finite. In this case, the growth rate $g(u)$ at small densities (small $u$ ) is responsible for the unbounded speed of spread. If $\alpha$ is close to 1 , this growth can be very weak $\left(g(\zeta) \sim \zeta^{\gamma}\right.$ with large $\left.\gamma\right)$ yet the speed of spread is still unbounded. If on the other hand $\alpha<1$, then large jumps tend to be more frequent, and jump sizes have unbounded mean. In this case, the growth rate for small densities is no longer the reason why the speed of spread is unbounded; in fact there may be no growth at all for small densities $(g(u)=0$ for small $u)$ for this to occur. Rather, the unbounded speed of spread results from growth that occurs solely for large densities $(g(\zeta)>0$ only for $\zeta \geq(\alpha-\rho) / 2 \alpha)$. The substance is transported towards $-\infty$ due large $(\alpha<1)$ negative jumps, resulting in an unbounded speed of spread. It is known that in this case, $(\alpha-\rho) / 2 \alpha$ is the probability that a jump is negative. If this fraction is large, then growth that occurs only for large densities, that is, $g(u)>0$ on $[(\alpha-\rho) / 2 \alpha, 1)$, already leads to an unbounded speed of spread. The case $\alpha=1$ is intermediate: any growth for small densities $(g(\zeta)>0$ for $\zeta>0)$ results in an unbounded speed of spread.

In the case $\alpha>1$, it would be interesting to know if the speed of spread is still unbounded if $\gamma \geq \alpha /(\alpha-1)$ or if a finite speed of spread occurs ((1.5) holds for large $c)$ if $\gamma$ becomes sufficiently large, that is, if growth is extremely weak for small densities $u$. In the case $\alpha<1$, it would be interesting to know if a finite speed of spread is possible at all if $g \geq 0$ and $g$ is not identically equal to 0 .

The main result in the case where $g$ is negative near $\zeta=0$ is the following.

Theorem 3.2. Let $u$ be the solution of (1.7) with initial data $u_{0}$ satisfying (3.1). Assume that $g^{\prime}(0)<$ 0 . Then there exists $c>0$ such that

$$
\limsup _{t \rightarrow \infty} \sup _{x \leq-c t} u(x, t)=0
$$

The result shows that negative proportional growth at small densities $\left(g^{\prime}(0)<0\right)$ always limits the speed of spread of a substance whose growth and spread are governed by (1.7), even for processes whose jump sizes tend to be very large $(\alpha<1)$. I am not aware of an interpretation of this result in the context of material science, similar to the use of (1.1) in [5].

The proofs will be given below. The main tools in the proofs are the comparison arguments given in the next section, together with the following crucial auxiliary result.

Lemma 3.3. Let $g \in C^{1}([0,1], \mathbb{R})$ and let $g_{0}, g_{1}$ be defined as in (2.11), depending on $\alpha$ and $\rho$.

(a) Let $\alpha \in(1,2)$. Suppose that $g(\zeta)>0$ for all $\zeta \in(0,1]$ and that there exist $c_{0}>0$ and $\gamma<\alpha /(\alpha-1)$ such that $g(\zeta) \geq c_{0} \zeta^{\gamma}$ for all $\zeta \in[0,1 / 2]$. Then given any $c>0$ there exists $\tau>0$ such that for all $\zeta \in[0,1]$

$$
g(\zeta) \geq c \tau^{-1 / \alpha} g_{0}(\zeta)+(\alpha \tau)^{-1} g_{1}(\zeta)
$$


International Journal of Differential Equations

(b) Let $\alpha=1$. Suppose that $g>0$ on $(0,1]$. Then given any $c>0$ there exists $\tau>0$ such that for all $\zeta \in[0,1]$

$$
g(\zeta) \geq c \tau^{-1} g_{0}(\zeta)+\tau^{-1} g_{1}(\zeta)
$$

(c) Let $\alpha \in(0,1)$. Suppose that $g \geq 0$ on $[0,1]$ and $g(\zeta)>0$ for all $\zeta \in[(\alpha-\rho) / 2 \alpha, 1)$. Then given any $c>0$ there exists $\tau>0$ such that for all $\zeta \in[0,1]$

$$
g(\zeta) \geq c \tau^{-1 / \alpha} g_{0}(\zeta)+(\alpha \tau)^{-1} g_{1}(\zeta)
$$

(d) Suppose that $g^{\prime}(0)<0$ and $g(\zeta)=0$ for $\zeta \in[1-\epsilon, 1]$ for some $\epsilon$. Then there exist $c \in \mathbb{R}$ and $\tau>0$ such that for all $\zeta \in[0,1]$

$$
g(\zeta) \leq c \tau^{-1 / \alpha} g_{0}(\zeta)+(\alpha \tau)^{-1} g_{1}(\zeta)
$$

Proof. Consider first statement (a). Let us write $F=F_{\alpha \rho}$ and $f=f_{\alpha \rho}$. Let $\alpha>1$ and let $M>0$ be large enough such that for some $c_{1}, c_{2}>0$ and all $x \leq-M$

$$
F(x) \geq c_{1}|x|^{-\alpha}, \quad f(x) \leq c_{2}|x|^{-1-\alpha} .
$$

This is possible by (2.6). Let $c>0$ be given, then we may increase $M$ further such that also

$$
c_{0} c_{1}^{\gamma} M^{r} \geq c_{2} \frac{\alpha-1}{\alpha} c^{\alpha /(\alpha-1)},
$$

where $r=(\alpha /(\alpha-1))-\gamma>0$. Now set $\delta=F(-M)$. Then for $0<\zeta=F(x) \leq \delta$, that is, $x<-M$, and for all $\tau \geq(M / c)^{\alpha /(\alpha-1)}$

$$
\begin{aligned}
c \tau^{-1 / \alpha} g_{0}(F(x))+(\alpha \tau)^{-1} g_{1}(F(x)) & =\left(c \tau^{-1 / \alpha}+(\alpha \tau)^{-1} x\right) f(x) \\
& \leq \frac{\alpha-1}{\alpha} c^{\alpha /(\alpha-1)}|x|^{1 /(1-\alpha)} f(x) \\
& \leq \frac{\alpha-1}{\alpha} c^{\alpha /(\alpha-1)}|x|^{1 /(1-\alpha)} c_{2}|x|^{-1-\alpha} \\
& =\frac{\alpha-1}{\alpha} c^{\alpha /(\alpha-1)} c_{2}|x|^{-\alpha^{2} /(\alpha-1)}
\end{aligned}
$$

where a standard calculus argument has been used to see that the expression $\left(c \tau^{-1 / \alpha}+\right.$ $\left.(\alpha \tau)^{-1} x\right)$ is maximal for $\tau=(|x| / c)^{\alpha /(\alpha-1)}$. We estimate further, using the choice of $M$,

$$
\begin{aligned}
c \tau^{-1 / \alpha} g_{0}(F(x))+(\alpha \tau)^{-1} g_{1}(F(x)) & \leq c_{0} c_{1}^{\gamma} M^{r}|x|^{-\alpha^{2} /(\alpha-1)} \\
& \leq c_{0} c_{1}^{\gamma}|x|^{r}|x|^{-\alpha^{2} /(\alpha-1)}=c_{0}\left(c_{1}|x|^{\alpha}\right)^{\gamma} \\
& \leq c_{0}(F(x))^{\gamma} \leq g(F(x)) .
\end{aligned}
$$


Therefore, for all $\tau \geq(M / c)^{\alpha /(\alpha-1)}$ and all $\zeta<\delta=F(-M)$,

$$
c \tau^{-1 / \alpha} g_{0}(\zeta)+(\alpha \tau)^{-1} g_{1}(\zeta) \leq g(\zeta)
$$

Since $g>0$ on $[\delta, 1]$ by assumption, this inequality can be achieved also on $[\delta, 1]$ by increasing $\tau$ even further. This proves part (a).

The proof of part (b) is straight forward: given $c>0$, note that $c g_{0}(\zeta)+g_{1}(\zeta) \leq 0$ on the interval $[0, F(-c)]$. Then $\left(c g_{0}(\zeta)+g_{1}(\zeta)\right) / \tau \leq g(\zeta)$ is true if $\tau$ is sufficiently large.

To prove part (c), let again $c>0$ be given. Let $u^{*}=\inf \{u \in[0,1] \mid g(u)>0\}$. Then $u^{*}<(\alpha-\rho) / 2 \alpha=F(0)$. Pick $\tau$ large enough such that $F\left(-c \alpha \tau^{1-1 / \alpha}\right)>u^{*}$. This is possible since $\alpha<1$. Then on $\left[0, u^{*}\right]$,

$$
c \tau^{-1 / \alpha} g_{0}(\zeta)+(\alpha \tau)^{-1} g_{1}(\zeta) \leq g(\zeta)
$$

since the left-hand side is nonpositive there by Proposition 2.1. By increasing $\tau$ further, we can obtain this estimate also for $\zeta \in\left[u^{*}, 1\right]$, using again that $g$ is assumed to be positive on $[(1-\beta) / 2,1]$.

To prove part $(\mathrm{d})$, note first that

$$
c \tau^{-1 / \alpha} g_{0}(\zeta)+(\alpha \tau)^{-1} g_{1}(\zeta) \geq g(\zeta)
$$

on an interval $[0, \delta]$ as soon as $\tau^{-1}+g^{\prime}(0)>0$, that is, for sufficiently small $\tau$. Increasing $c$ sufficiently and noting that $g=0$ near $\zeta=1$ extends this inequality to the entire interval $[0,1]$.

Proof of Theorem 3.1. The proof uses the same argument for all three parts; so we give details only in part (a). Let $\epsilon>0$. We replace $u$ with $\tilde{u}=(1+\epsilon) u$ and $g$ with $\tilde{g}$, where $\tilde{g}(\zeta)=$ $(1+\epsilon) g\left((1+\epsilon)^{-1} \zeta\right)$. Then

$$
\partial_{t} \tilde{u}+A \tilde{u}=\tilde{g}(\tilde{u}) .
$$

Then $\tilde{g}(\zeta) \geq \tilde{c}_{0} \zeta^{\gamma}$ for $\zeta \in[0,1 / 2]$, possibly with a changed $c_{0}$, and additionally $\tilde{g}>0$ on $(0,1]$. Let $c>0$ be given, then there exists $\tau>0$ such that

$$
\widetilde{g}(\zeta) \geq(c+1) \tau^{-1 / \alpha} g_{0}(\zeta)+(\alpha \tau)^{-1} g_{1}(\zeta)
$$

for all $\zeta \in[0,1]$ by Lemma 3.3. By extending $g_{0}$ and $g_{1}$ to be zero on $[1,1+\epsilon]$, this inequality is true on $[0,1+\epsilon]$. Now find a constant $d$ such that $v_{0}(x)=H(x-d) \leq \tilde{u}(x, 0)$ for all $x$. This is possible since $\lim _{x \rightarrow \infty} \tilde{u}(x, 0)=1+\epsilon$. By Proposition 4.3, we see that

$$
\tilde{u}(x, t) \geq F\left((x-d) t^{-1 / \alpha}\right)
$$


for all $x \in \mathbb{R}, t>0$. This is in particular true for $t=\tau$. Now use Proposition 4.1 and (3.19) to infer that

$$
\tilde{u}(x, t) \geq F\left((x-d+(c+1) t) \tau^{-1 / \alpha}\right)
$$

for all $x \in \mathbb{R}, t \geq \tau$. Therefore for $t \geq \tau$ and $x \geq-c t$,

$$
\begin{aligned}
\tilde{u}(x, t) & \geq F\left((x-d+(c+1) t) \tau^{-1 / \alpha}\right) \\
& \geq F\left((-c t-d+(c+1) t) \tau^{-1 / \alpha}\right) \\
& =F\left((t-d) \tau^{-1 / \alpha}\right) .
\end{aligned}
$$

As $t \rightarrow \infty$, the right-hand side goes to 1 . Rewriting this in terms of $u$, we see that

$$
\liminf _{t \rightarrow \infty} \inf _{x \geq-c t} \tilde{u}(x, t) \geq(1+\epsilon)^{-1} .
$$

Since $\epsilon>0$ was arbitrary, the desired result follows.

In case of part (b), the same argument can be used without changes, appealing to part (b) of Lemma 3.3.

In case of part (c), we have to restrict $\epsilon$ such that $\tilde{g}>0$ on $[(\alpha-\rho) / 2 \alpha, 1]$, that is, $g>0$ on $[(\alpha-\rho) /(2 \alpha(1+\epsilon)), 1]$. The rest of the proof is again unchanged, using part (c) of Lemma 3.3.

Proof of Theorem 3.2. We replace $u$ with $\tilde{u}=(1 / 2) u$ and $g$ with $\tilde{g}$, where $\tilde{g}(\zeta)=(1 / 2) g(2 \zeta)$ for $0 \leq \zeta \leq 1 / 2$ and $\tilde{g}(\zeta)=0$ for $\zeta \in(1 / 2,1]$. Then $\tilde{g}^{\prime}(0)=g^{\prime}(0)<0$ and

$$
\partial_{t} \tilde{u}+A \tilde{u}=\tilde{g}(\tilde{u}) .
$$

By Lemma 3.3, part (d), there exist $c>0$ and $\tau>0$ such that

$$
\tilde{g}(\zeta) \leq(c-1) \tau^{-1 / \alpha} g_{0}(\zeta)+(\alpha \tau)^{-1} g_{1}(\zeta)
$$

Since $\lim _{x \rightarrow \infty} \tilde{u}(x, 0)=1 / 2$ and $u(x, 0)=0$ for $x<0$, we can find $d>0$ such that $F(x+$ d) $\left.\tau^{-1 / \alpha}\right) \geq \widetilde{u}(x, 0)$ for all $x \in \mathbb{R}$. Using Proposition 4.1 , one sees that

$$
F\left((x+d+(c-1) t) \tau^{-1 / \alpha}\right) \geq \tilde{u}(x, t)
$$

for all $x \in \mathbb{R}, t>0$. Therefore for $t>0$ and $x \leq-c t$,

$$
\begin{aligned}
\tilde{u}(x, t) & \leq F\left((x+d+(c-1) t) \tau^{-1 / \alpha}\right) \\
& \leq F\left((-c t+d+(c-1) t) \tau^{-1 / \alpha}\right) \\
& =F\left((-t+d) \tau^{-1 / \alpha}\right) .
\end{aligned}
$$


The right-hand side tends to 0 as $t \rightarrow \infty$. In terms of $u$, this implies

$$
\limsup _{t \rightarrow \infty} \sup _{x \leq-c t} u(x, t)=0 .
$$

This concludes the proof.

\section{Facts about Fractional Reaction-Diffusion Equations}

In this section we summarize some basic theory about (1.7) that is needed in this note. A broader and deeper discussion may be found in [10].

We work in the Banach space

$$
\mathcal{C}_{\lim }=\left\{w \in C(\mathbb{R}) \mid \lim _{x \rightarrow \infty} w(x) \text { and } \lim _{x \rightarrow-\infty} w(x) \text { exist }\right\}
$$

equipped with the supremum norm $\|\cdot\|$. Let $A=A_{\alpha \rho}$ be the pseudodifferential operator with symbol (1.8) and parameters $\alpha, \rho$, with $0<\alpha<2$ and $|\rho|<\min \{\alpha, 2-\alpha\}$. Subscripts $\alpha, \rho$ will again frequently be suppressed. Solutions of the free equation (2.1) with initial data $u(\cdot, 0)=\varphi \in \mathcal{C}_{\lim }$ then can be written in terms of the fundamental solution given in (2.2), namely,

$$
u(x, t)=\int_{\mathbb{R}} W_{\alpha \rho}(x-y, t) \varphi(y) d y .
$$

For fixed $\alpha$ and $\rho$ and $\varphi \in \mathcal{C}_{\text {lim }}$, we therefore define

$$
S(t) \varphi(x)=u(x, t)
$$

where $u$ is given by (4.2). Then $S(t)_{t \geq 0}$ is a positive $C_{0}$ semigroup on $\mathcal{C}_{\lim }$ and a Feller semigroup on the subspace of functions in $C_{\lim }$ that vanish at $\pm \infty$. If $\psi$ is a continuous function from $[0, T]$ to $\mathcal{C}_{\lim }$, then solutions of the inhomogeneous equation

$$
\partial_{t} u(x, t)+A u(x, t)=\psi(x, t), \quad u(\cdot, 0)=\varphi
$$

can be written with the variation-of-constants formula:

$$
u(\cdot, t)=S(t) \varphi+\int_{0}^{t} S(t-s) \psi(\cdot, s) d s
$$

A continuous curve $u:[0, T] \rightarrow \mathcal{C}_{\lim }$ that satisfies (4.5) is commonly called a mild solution of (4.4). Next let $g:[0, \infty) \times \mathbb{R} \rightarrow \mathbb{R}$ be locally Lipschitz continuous in both variables and let $\varphi \in \mathcal{C}_{\lim }$. Then the equation $\partial_{t} u(x, t)+A u(x, t)=g(t, u(x, t))$ (for which (1.7) is a special case) has a unique mild solution $u \in C\left([0, T), C_{\lim }\right)$, where $0<T \leq \infty$ is maximal. Either $T=\infty$, or 
$\|u(\cdot, t)\| \rightarrow \infty$ as $t \uparrow T$. The solution can be obtained as the locally in time uniform limit of the iteration scheme:

$$
u_{n+1}(\cdot, t)=S(t) \varphi+\int_{0}^{t} S(t-s) g\left(s, u_{n}(\cdot, s)\right) d s \quad(n=0,1, \ldots)
$$

with $u_{0}$ being arbitrary, for example, $u_{0}(\cdot, t)=S(t) \varphi$. It is possible to set up a more general solution theory, but this is not needed for the purposes of this paper.

Solutions of (1.7) satisfy comparison theorems. Results of this type are true for all Feller semigroup. A systematic study of such semigroups and their generators was carried out in [22], following the seminal work on this topic in [23]. For the sake of completeness, a comparison result is stated here, and its proof is sketched.

Proposition 4.1. Let $u, v \in C\left([0, T], \mathcal{C}_{\lim }\right)$ be mild solutions of the equations

$$
\partial_{t} u+A u=g(u), \quad \partial_{t} v+A v=h(v),
$$

where $g, h: \mathbb{R} \rightarrow \mathbb{R}$ are locally Lipschitz continuous. If

$$
\begin{gathered}
g(\zeta) \leq h(\zeta), \quad \forall \zeta \in \mathbb{R}, \\
u(\cdot, 0) \leq v(\cdot, 0),
\end{gathered}
$$

then

$$
u(x, t) \leq v(x, t) \quad \forall(x, t) \in \mathbb{R} \times[0, T]
$$

Proof. Let $M=\max _{[0, T]}(\|u(\cdot, t)\|+\|v(\cdot, t)\|+1)$. Let $\lambda>\left|g^{\prime}(\zeta)\right|+\left|h^{\prime}(\zeta)\right|$ for all $|\zeta| \leq M$. Without loss of generality we may assume that $g$ and $h$ are constant outside $[-M, M]$. Set

$$
U(x, t)=e^{\lambda t} u(x, t), \quad V(x, t)=e^{\lambda t} v(x, t),
$$

and observe that $U$ and $V$ satisfy

$$
\begin{aligned}
& \partial_{t} U+A U=\tilde{g}(t, U), \\
& \partial_{t} V+A V=\tilde{h}(t, V)
\end{aligned}
$$

with $\tilde{g}(t, \zeta)=\lambda \zeta+e^{\lambda t} g\left(e^{-\lambda t} \zeta\right)$ and $\tilde{h}(t, \zeta)$ defined similarly. Clearly, $\tilde{g}(t, \zeta) \leq \tilde{h}(t, \zeta)$ for all $\zeta$. The function $\tilde{g}$ is nondecreasing in its second argument, since for almost all $\zeta$

$$
\partial_{\zeta} \tilde{g}(t, \zeta)=\lambda+g^{\prime}\left(e^{-\lambda \zeta}\right) \geq 0 .
$$

Consider the iteration scheme:

$$
U_{n+1}(\cdot, t)=S(t) u(\cdot, 0)+\int_{0}^{t} S(t-s) \tilde{g}\left(s, U_{n}(\cdot, s)\right) d s
$$


and similarly for $V_{n}$ and $\tilde{h}$. The scheme for the $U_{n}$ converges to the limit $U$, and the scheme for the $V_{n}$ converges to the limit $V$.

We now employ a standard induction argument to show that $U_{n} \leq V_{n}$ on $\mathbb{R} \times[0, T]$ for all $n$. Let $U_{0}(\cdot, t)=S(t) u(\cdot, 0)$ and $V_{0}(\cdot, t)=S(t) v(\cdot, 0)$, then $U_{0} \leq V_{0}$ on $\mathbb{R} \times[0, T]$ since $S$ is a positive semigroup and $u(\cdot, 0) \leq v(\cdot, 0)$. Suppose $U_{n} \leq V_{n}$ on $\mathbb{R} \times[0, T]$, then

$$
\begin{aligned}
U_{n+1}(\cdot, t) & =S(t) u(\cdot, 0)+\int_{0}^{t} S(t-s) \tilde{g}\left(s, U_{n}(\cdot, s)\right) d s \\
& \leq S(t) v(\cdot, 0)+\int_{0}^{t} S(t-s) \tilde{g}\left(s, V_{n}(\cdot, s)\right) d s \\
& \leq S(t) v(\cdot, 0)+\int_{0}^{t} S(t-s) \tilde{h}\left(s, V_{n}(\cdot, s)\right) d s \\
& =V_{n+1}(\cdot, t),
\end{aligned}
$$

which completes the induction step. Taking the limit, this implies that $U \leq V$ and therefore also $u \leq v$ on $\mathbb{R} \times[0, T]$. This proves the proposition.

Corollary 4.2. Consider a mild solution $u \in C\left([0, T), \mathcal{C}_{\lim }\right)$ of $(1.7)$ and assume that $g$ is locally Lipschitz continuous. If $g(\gamma) \geq 0$ for some $\gamma$ and $u(\cdot, 0) \geq \gamma$, then $u(\cdot, t) \geq \gamma$ for all $t$. If $g(\gamma) \geq$ $0 \geq g(\delta)$ for some $\gamma<\delta$ and $\gamma \leq u(\cdot, 0) \leq \delta$, then $\gamma \leq u(\cdot, t) \leq \delta$ for all $t$, and the solution can be continued to $\mathbb{R} \times[0, \infty)$.

The proof consists in observing that the constant functions $v(x, t)=\gamma$ and $w(x, t)=$ $\delta$ solve (1.7) with right-hand sides 0 and therefore must be pointwise bounds for the solution, by Proposition 4.1. If the solution remains bounded between two constants, then its supremum norm remains bounded and it can be continued to $\mathbb{R} \times[0, \infty)$.

Also required is a comparison result for solutions whose initial data are step functions. Since such initial data are not in $\mathcal{C}_{\text {lim }}$, a separate argument is required.

Proposition 4.3. Let $u \in C\left([0, T], \mathcal{C}_{\lim }\right)$ be a mild solution of (1.7), with locally Lipschitz continuous g. Assume that

$$
u(x, 0) \geq v_{0}(x)=a_{0}+\sum_{j=1}^{N} a_{j} H\left(x-c_{j}\right) \quad \forall x \in \mathbb{R},
$$

where $a_{j} \in \mathbb{R}, c_{1}<c_{2} \cdots<c_{N}$, and $H$ is the Heaviside function. Let $\gamma=\min _{\mathbb{R}} v_{0}(x)$ and $\delta=$ $\max _{\mathbb{R}} v_{0}(x)$. Assume also that $g \geq 0$ on $[\gamma, \delta]$. Then

$$
u(x, t) \geq a_{0}+\sum_{j=1}^{N} a_{j} V_{\alpha \rho}\left(x-c_{j}, t\right) \quad \forall x \in \mathbb{R}, 0<t \leq T,
$$

where $V_{\alpha \rho}$ is defined in (2.4). 
Proof. We know that $u(x, t) \geq \gamma$ and thus may assume that $g(\zeta) \geq 0$ also for $\zeta<\gamma$. For arbitrary $\epsilon, \sigma>0$, we set

$$
v_{\epsilon \sigma}(x)=\frac{1}{\sigma} \int_{0}^{\sigma} v_{0}(x-z) d z-\epsilon .
$$

Then $v_{\epsilon \sigma}$ is piecewise linear and constant outside the interval $\left[c_{1}, c_{N}+\sigma\right]$; in particular, $v_{\epsilon \sigma} \in$ $\mathcal{C}_{\text {lim. }}$. Solving (2.1) with initial data $v_{\epsilon, \sigma}$ gives the solution

$$
G_{\epsilon \sigma}(x, t)=\frac{1}{\sigma} \int_{0}^{\sigma} \sum_{j=1}^{N} a_{j} V_{\alpha \rho}\left(x-z-c_{j}, t\right)+a_{0}-\epsilon .
$$

Given $\epsilon>0$, it is possible to find $\sigma>0$ such that $v_{\epsilon \sigma}<u_{0}(x)$ on $\mathbb{R}$, since $u_{0}$ is uniformly continuous. Clearly, $\gamma-\epsilon \leq G_{\epsilon \sigma} \leq \delta$. We may therefore view $G_{\epsilon \sigma}$ as a solution of (1.7) with a right-hand side $h$ that satisfies $h(\zeta)=0 \leq g(\zeta)$ for $\zeta \leq \delta$ and $h(\zeta) \leq g(\zeta)$ also for $\zeta>\delta$. Then by Proposition 4.1

$$
u(x, t) \geq G_{\epsilon, \sigma}(x, t) \quad \forall x \in \mathbb{R}, 0<t \leq T .
$$

Send $\delta$ to 0 , then since $V_{\alpha \rho}$ is uniformly continuous, (4.16) is obtained with $a_{0}$ replaced by $a_{0}-\epsilon$ on the right-hand side. Now send $\epsilon$ to 0 and (4.16) follows.

\section{Conclusion}

In this note, conditions for the speed of spread of solutions of fractional scalar reactiondiffusion equations to be finite or infinite have been derived. If the reaction term is positive for all positive arguments, then this speed is shown to be infinite as soon as the reaction term describes some very weak growth for low densities. This is in contrast to the corresponding problem for standard diffusion, where the speed of spread is always finite for such reaction terms. On the other hand, if the reaction term is negative for small positive arguments, then the speed of spread is finite, just as it is for the case of standard diffusion.

\section{References}

[1] A. N. Kolmogorov, I. G. Petrovskii, and N. S. Piskunov, "Étude de l'équation de diffusion avec accroissement de la quantité de matière, et son application à un probleme biologique," Bulletin Université d'Etat à Moscou, vol. 17, pp. 1-26, 1937.

[2] H. P. McKean, "Application of Brownian motion to the equation of Kolmogorov-PetrovskiiPiskunov," Communications on Pure and Applied Mathematics, vol. 28, no. 3, pp. 323-331, 1975.

[3] C. R. Doering, C. Mueller, and P. Smereka, "Interacting particles, the stochastic Fisher-KolmogorovPetrovsky-Piscounov equation, and duality," Physica A, vol. 325, no. 1-2, pp. 243-259, 2003.

[4] D. G. Aronson and H. F. Weinberger, "Multidimensional nonlinear diffusion arising in population genetics," Advances in Mathematics, vol. 30, no. 1, pp. 33-76, 1978.

[5] S. M. Allen and J. W. Cahn, "A microscope theory for antiphase boundary motion and its application to antiphase domain coarsening," Acta Materialia, vol. 27, no. 6, pp. 1085-1095, 1979.

[6] U. Ebert and W. V. van Saarloos, "Front propagation into unstable states: universal algebraic convergence towards uniformly translating pulled fronts," Physica D, vol. 146, no. 1-4, pp. 1-99, 2000. 
[7] J. Fort and T. Pujol, "Progress in front propagation research," Reports on Progress in Physics, vol. 71, no. 8, 41 pages, 2008.

[8] F. Mainardi, Y. Luchko, and G. Pagnini, "The fundamental solution of the space-time fractional diffusion equation," Fractional Calculus \& Applied Analysis, vol. 4, no. 2, pp. 153-192, 2001.

[9] K. Sato, Levy Processes and Infinitely Divisible Distributions, Cambridge University Press, Cambridge, UK, 1999.

[10] B. Baeumer, M. Kovács, and M. M. Meerschaert, "Fractional reproduction-dispersal equations and heavy tail dispersal kernels," Bulletin of Mathematical Biology, vol. 69, no. 7, pp. 2281-2297, 2007.

[11] D. del-Castillo-Negrete, B. A. Carreras, and V. E. Lynch, "Front dynamics in reaction-diffusion systems with Levy flights: a fractional diffusion approach," Physical Review Letters, vol. 91, no. 1, Article ID 018302, 2003.

[12] R. Mancinelli, D. Vergni, and A. Vulpiani, "Superfast front propagation in reactive systems with nonGaussian diffusion," Europhysics Letters, vol. 60, no. 4, pp. 532-538, 2002.

[13] R. Mancinelli, D. Vergni, and A. Vulpiani, "Front propagation in reactive systems with anomalous diffusion," Physica D, vol. 185, no. 3-4, pp. 175-195, 2003.

[14] D. H. Zanette, "Wave fronts in bistable reactions with anomalous Lévy-flight diffusion," Physical Review E, vol. 55, no. 1, supplement B, pp. 1181-1184, 1997.

[15] G. M. Zaslavsky, "Chaos, fractional kinetics, and anomalous transport," Physics Reports, vol. 371, no. 6, pp. 461-580, 2002.

[16] K. W. Schaaf, "Asymptotic behavior and traveling wave solutions for parabolic functional-differential equations," Transactions of the American Mathematical Society, vol. 302, no. 2, pp. 587-615, 1987.

[17] B. Baeumer, M. Kovács, and M. M. Meerschaert, "Numerical solutions for fractional reaction-diffusion equations," Computers E Mathematics with Applications, vol. 55, no. 10, pp. 2212-2226, 2008.

[18] X. Cabre and J.-M. Roquejoffre, "Propagation de fronts dans les équations de Fisher-KPP avec diffusion fractionnaire," http://arxiv.org/abs/0905.1299.

[19] Y. Nec, A. A. Nepomnyashchy, and A. A. Golovin, "Front-type solutions of fractional Allen-Cahn equation," Physica D, vol. 237, no. 24, pp. 3237-3251, 2008.

[20] J. Nolan, Stable Distributions-Models for Heavy Tailed Data, chapter 1, Birkhäuser, Boston, Mass, USA, 2010.

[21] V. M. Zolotarev, One-Dimensional Stable Distributions, vol. 65 of Translations of Mathematical Monographs, American Mathematical Society, Providence, RI, USA, 1986, translated from Odnomernye Ustoichioye Raspredelniia, Nauka, Moscow, Russia, 1982.

[22] J.-M. Bony, P. Courrège, and P. Priouret, "Semi-groupes de Feller sur une variété à bord compacte et problèmes aux limites intégro-différentiels du second ordre donnant lieu au principe du maximum," Annales de l'Institut Fourier, vol. 18, pp. 369-521, 1968.

[23] W. von Waldenfels, "Positive Halbgruppen auf einem n-dimensionalen Torus," Archiv für Mathematische Logik und Grundlagenforschung, vol. 15, pp. 191-203, 1964. 


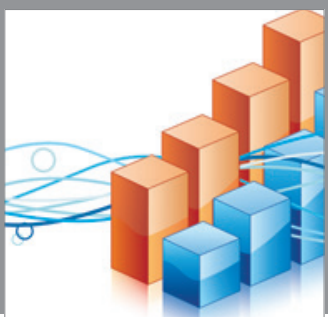

Advances in

Operations Research

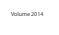

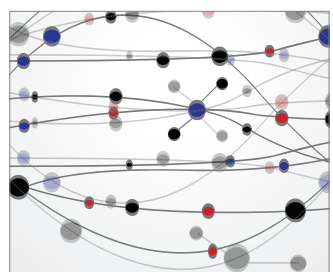

\section{The Scientific} World Journal
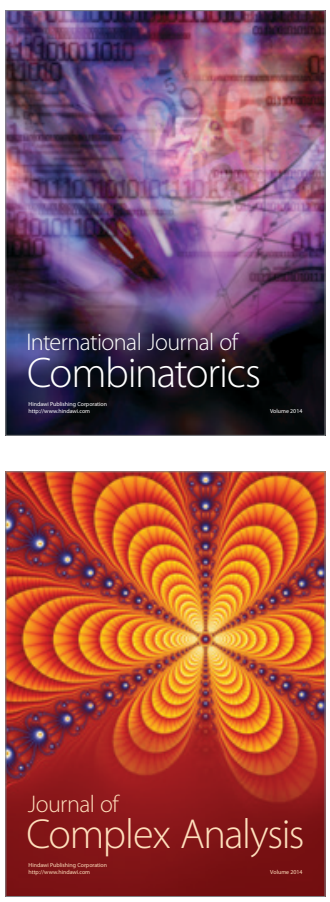

International Journal of

Mathematics and

Mathematical

Sciences
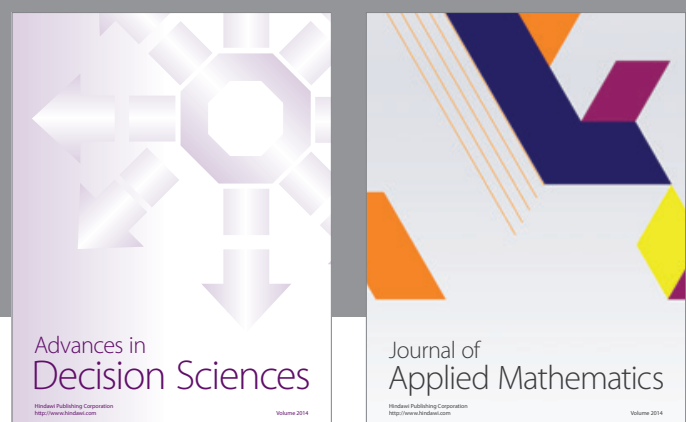

Journal of

Applied Mathematics
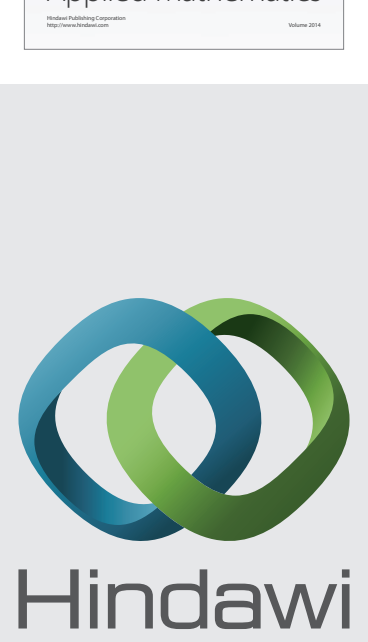

Submit your manuscripts at http://www.hindawi.com
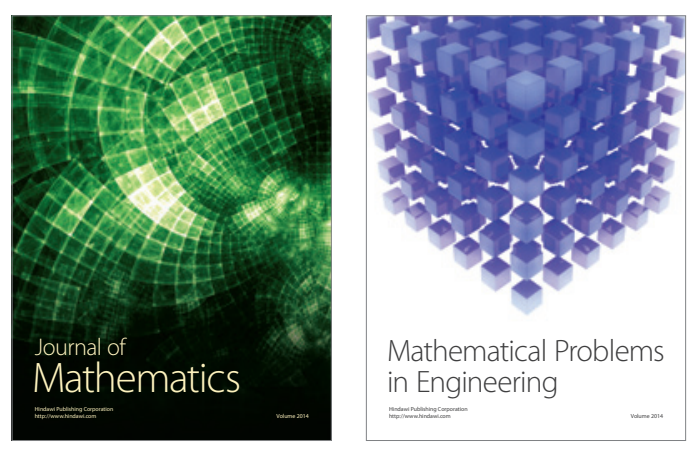

Mathematical Problems in Engineering
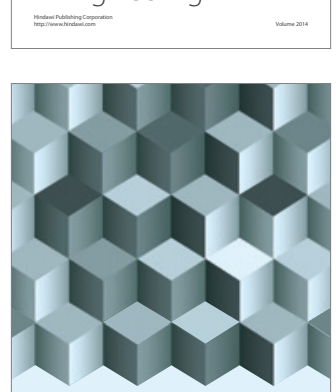

Journal of

Function Spaces
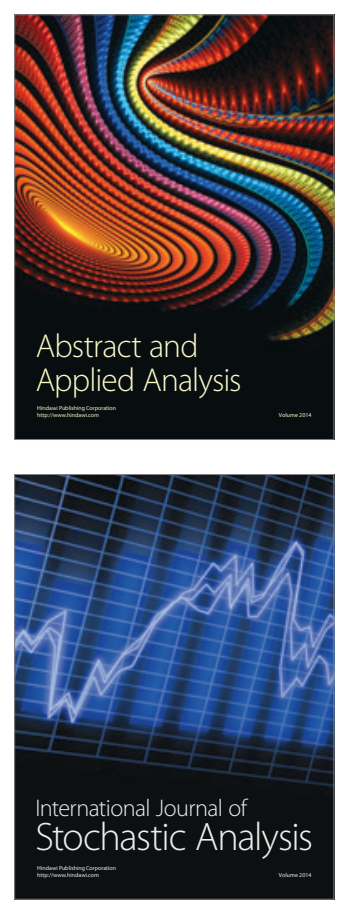

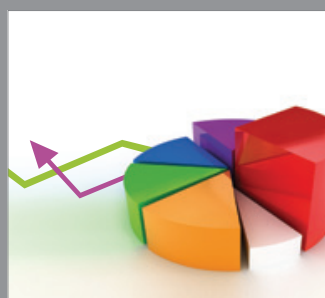

ournal of

Probability and Statistics

Promensencen
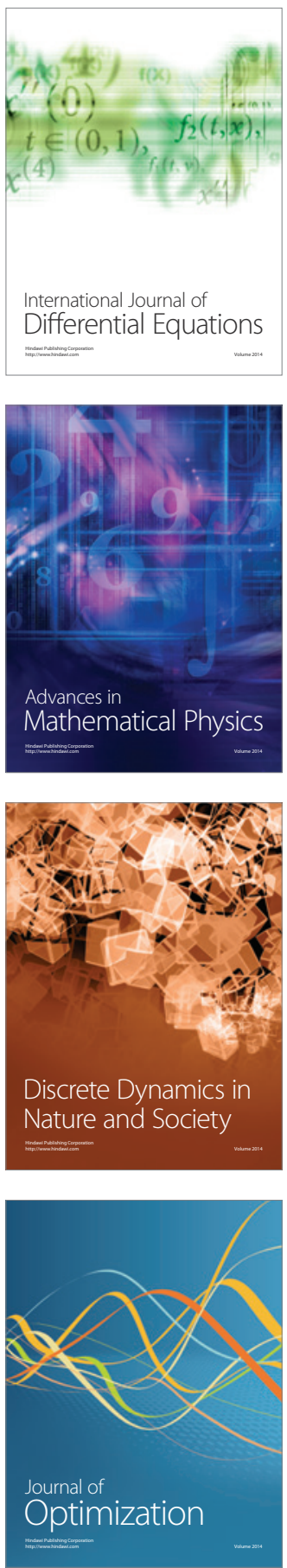